\title{
Clinical Spectrum and Treatment Outcomes of HIV Associated Cancers in a Tertiary Care Centre
}

\author{
Senthilkumar Elumalai¹, Kalaichelvi Kannan², Sureshkumar Sundarrajan³, Raja Gopal
}

${ }^{1}$ Department of Medical Oncology, Madras Medical College, Chennai, Tamilnadu, India. ${ }^{2}$ Department of Medical Oncology, Madras Medical College, Chennai, Tamilnadu, India. ${ }^{3}$ Department of Medical Oncology, Madras Medical College, Chennai, Tamilnadu, India. ${ }^{4}$ Department of Medical Oncology, Madras Medical College, Chennai,

\author{
Tamilnadu, India.
}

\section{ABSTRACT}

\section{BACKGROUND}

India has a high adult HIV prevalence rate $(0.26 \%)$. In the Highly Active Anti Retro Viral Therapy (HAART) era, there is increased lifespan of HIV patients which confers a higher lifetime risk of developing cancer. The estimated prevalence of HIV infection in the age group 15-49 years in India is $0.26 \%$ according to the 2015 NACO (National AIDS Control Organisation) report. Patients with HIV infection are prone to develop malignancies, especially viral mediated cancers like Kaposi sarcoma (related to HHV-8), invasive cervical cancers (HPV related) and aggressive B cell lymphomas (often EBV related) due to the loss of immune surveillance. Cancers are an important cause of morbidity and mortality in patients with HIV infection accounting for nearly $36 \%$ of the deaths in PLWHA (People Living with HIV-AIDS).

\section{METHODS}

A retrospective analysis of the patients with HIV and cancer registered in the Department of Medical Oncology, during January 2014 to June 2018 was done using baseline patient factors, pathology reports and treatment details. The survival analysis was done using IBM SPSS software (v-21).

\section{RESULTS}

The study included 44 patients with HIV and cancer, all of whom were on antiretroviral therapy. The mean time to presentation of cancer after diagnosis of AIDS was 5.4 years. Non-AIDS Defining Cancers (NADC) were present in $72.7 \%$ of patients. The most common malignancy was Non-Hodgkin`s lymphoma (31.8\%), plasmablastic lymphoma being the most common subtype. Extranodal presentations (78\%) were more common. B symptoms were present in $57 \%$ of cases. Head \& neck, penile, anal, cervical and colorectal cancers were the common solid tumours. Majority of the NHL patients presented with stage 1 disease, while most solid tumours were in advanced stages. Among the 14 NHL patients, 7 received chemotherapy and had a median survival of 6 months. Chemotherapy regimens included CHOP, CVP, R-CHOP and R-CHOP-E. Treatment schedule was completed in 4 patients with 2 achieving partial response. $46.6 \%$ of HIV patients with solid tumours received chemotherapy with 5 achieving partial response (PR) and 1 achieving complete response (CR). They had a median survival of 7 months as compared to 2 months in those who received only supportive care.

\section{CONCLUSIONS}

In the HAART era, NADCs are more common than ADCs. The most common malignancy in HIV patients in this study is NHL, with plasmablastic subtype being the commonest. In the HIV associated lymphoma patients, chemotherapy provides a response rate of $50 \%$ and achieves a median survival of 6 months as compared to only 1.5 months in those treated with supportive care. Standard chemotherapy in HIV patients with solid cancers achieve a response rate of $46 \%$.

\section{KEY WORDS}

HIV, Cancer, Non-AIDS Defining Cancers (NADC), AIDS Defining Cancers (ADC), HAART, PLWHA (People Living with HIV-AIDS)
Corresponding Author:

Dr. Senthilkumar Elumalai,

Assistant Professor,

Department of Medical Oncology

Madras Medical College,

Rajiv Gandhi Government General Hospital Chennai 600003

E-mail: esenthilkmc02@gmail.com

DOI: $10.14260 /$ jemds/2019/810

Financial or Other Competing Interests: None.

How to Cite This Article:

Elumalai S, Kannan K, Sundarrajan, $S$, et al. Clinical spectrum and treatment outcomes of HIV associated cancers in a tertiary care centre. J. Evolution Med. Dent. Sci. 2019;8(50):3739-3743, DOI: $10.14260 /$ jemds $/ 2019 / 810$

Submission 03-07-2019,

Peer Review 27-11-2019,

Acceptance 04-12-2019,

Published 16-12-2019. 


\section{BACKGROUND}

India has a high adult HIV prevalence rate $(0.26 \%)$. In the Highly Active Anti Retro Viral Therapy (HAART) era, there is increased lifespan of HIV patients which confers a higher lifetime risk of developing cancer. The estimated prevalence of HIV infection in the age group 15-49 years in India is $0.26 \%$ according to the 2015 NACO (National AIDS Control Organisation) report.[1] Patients with HIV infection are prone to develop malignancies, ${ }^{2}$ especially viral mediated cancers like Kaposi sarcoma (related to HHV-8), invasive cervical cancers (HPV related) and aggressive B cell lymphomas (often EBV related) due to the loss of immune surveillance. Cancers are an important cause of morbidity and mortality in patients with HIV infection accounting for nearly $36 \%$ of the deaths in PLWHA (People Living with HIV-AIDS). ${ }^{3}$ With the advent of highly active retroviral therapy (HAART) in the nineties, patients live longer with improved CD4 cell counts and controlled viral loads. In the pre-HAART era, the AIDS defining cancers (ADC) like aggressive Non-Hodgkin`s lymphoma, Kaposi`s sarcoma, primary CNS lymphomas and invasive cervical cancers constituted the majority of cancers in PLWHA patients. However, in the HAART era, Non-AIDS defining cancers (NADC) are more common than AIDS defining cancers.[4,5] The effective antiviral regimens also allow for the patients to receive more intensive regimens resulting in better outcomes. The purpose of this study is to analyse the clinical spectrum and treatment outcomes of patients with HIV and cancer.

We wanted to assess the pattern of cancers, clinical profile and the treatment outcomes in the cancer patients with HIV.

\section{METHODS}

The study was a retrospective observational study which included all the patients with HIV and cancer attending the Medical Oncology Department OPD between January 2014 and June 2018. Data obtained from the case records was used to fill up the proforma which included the patient factors (Age, sex, performance status, BMI, substance abuse), HIV related parameters (Time since diagnosis of HIV, baseline CD4 counts, comorbid conditions, treatment with HAART), tumour related factors (Site, type of cancer, subtype, stage, prognostic factors depending on the histology) and treatment aspects (Type of treatment, any requirement of dose modifications, toxicity, response, compliance).Ethical committee clearance was obtained. An informed consent was obtained from all patients. Cancers were diagnosed and classified based on histopathology reports. The AIDS defining cancers were invasive cervical cancers, Non-Hodgkin`s lymphoma (Including primary CNS lymphoma, primary effusion lymphomas) and Kaposi's sarcoma. Other cancers are considered to be Non-AIDS defining cancers. For incomplete case records, the pending data was collected from concerned labs and patient's relatives were contacted for other relevant information.

\section{Statistical Analysis}

Statistical analysis was done using IBM SPSS software (v-21). Categorical values were summarized in terms of frequencies and proportions. Descriptive analysis was done for continuous variables using mean and standard deviations. Kaplan Meier curves were used for comparison of survival between patient subgroups.

\section{RESULTS}

The study included 44 patients with HIV and cancer (17 female patients and 27 male patients) with an average age of 42 years. A majority of the patients (71\%) were in the $31-50$ years age group, as shown in Fig. 1.

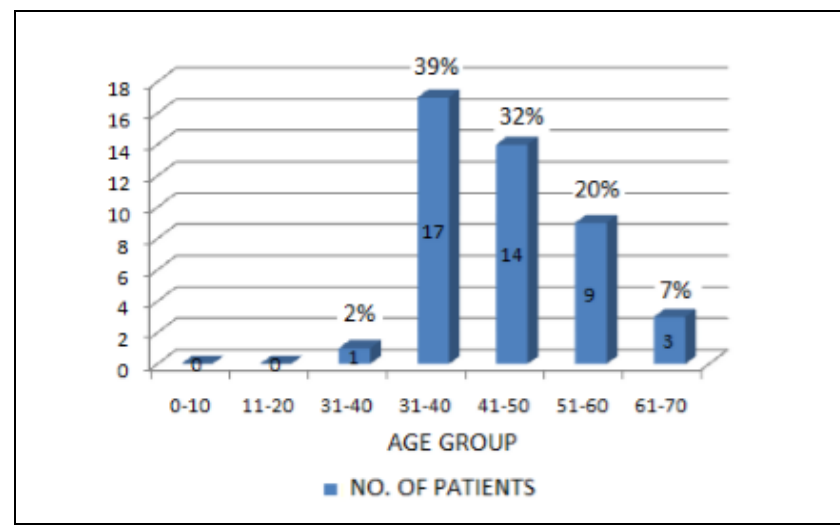

Figure 1. Age Wise Distribution of the Patients with HIV and Cancer
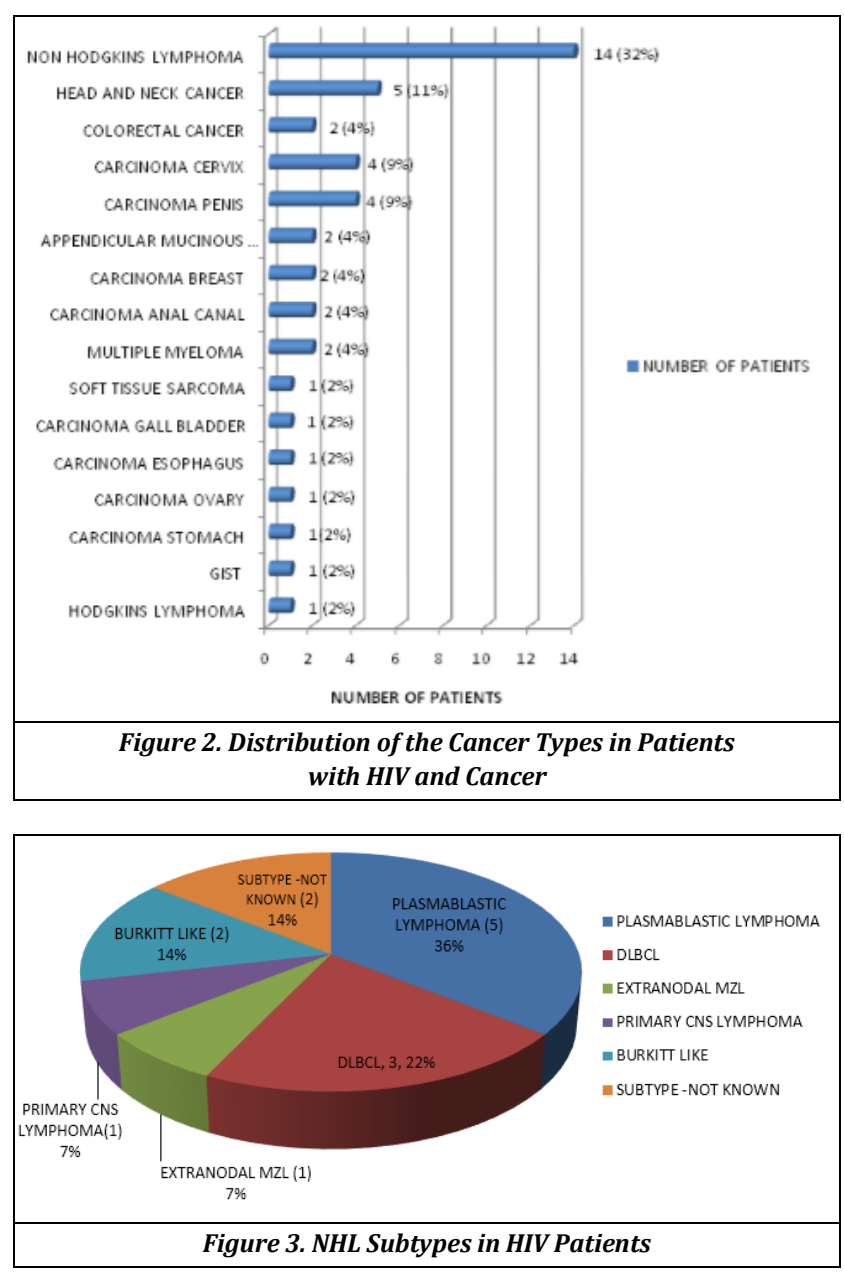

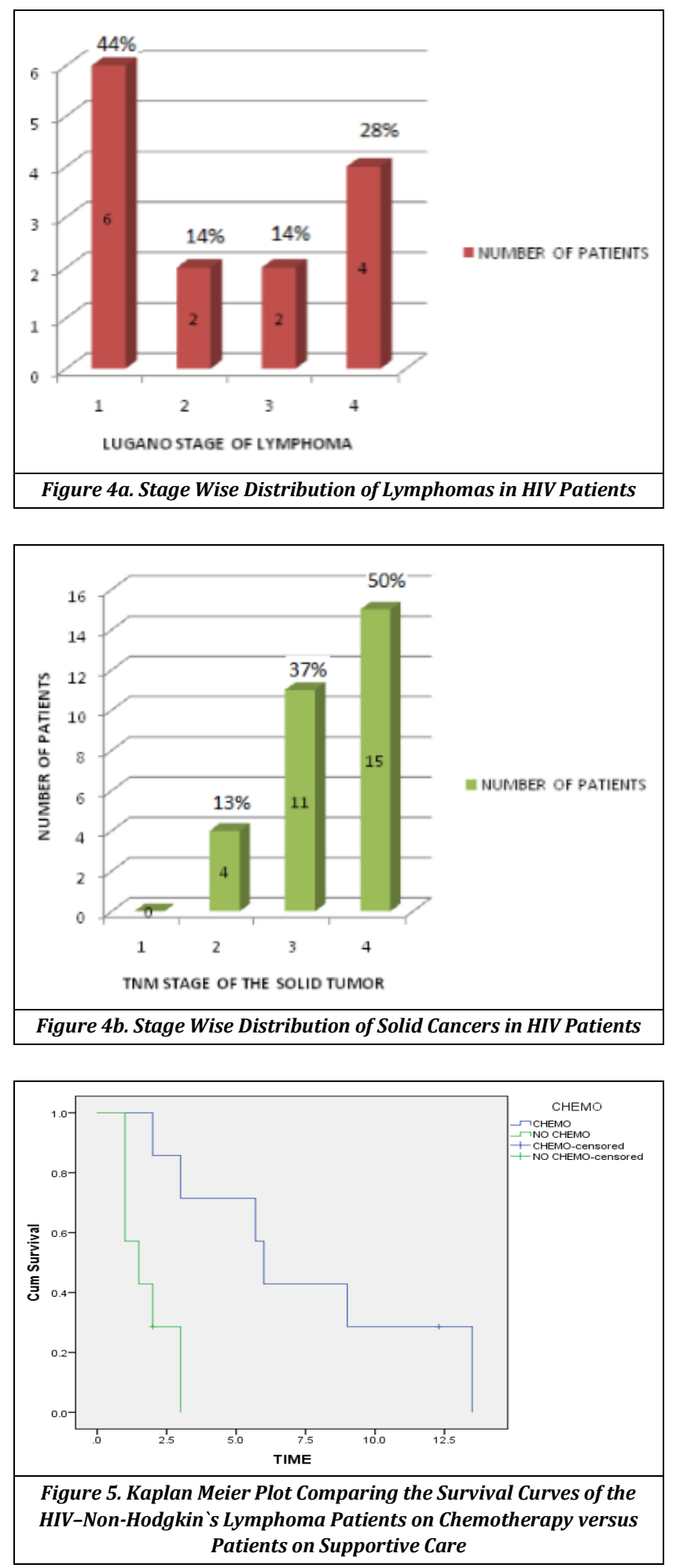

The mean BMI of the study group was 19.45 (Range: 10 26.5). Thirteen of the forty-four patients (29.5\%) had poor general condition with a performance status of 3 or 4 by the ECOG (Eastern Cooperative Oncology Group) scale. All patients were already enrolled for anti-retroviral therapy. The mean time to presentation of cancer after the diagnosis of HIV infection was 5.4 years (Range: 2 months to 15 years). Thirty-two patients had Non-AIDS Defining Cancers (NADC) and twelve had AIDS defining cancers (ADC). The most common malignancy seen was Non-Hodgkin`s lymphoma, accounting for fourteen cases. None of the patients had Kaposi's sarcoma. Head \& neck cancers, carcinoma penis, anal cancer, cervical cancer and colorectal cancers were the common solid tumours as shown in Fig. 2.

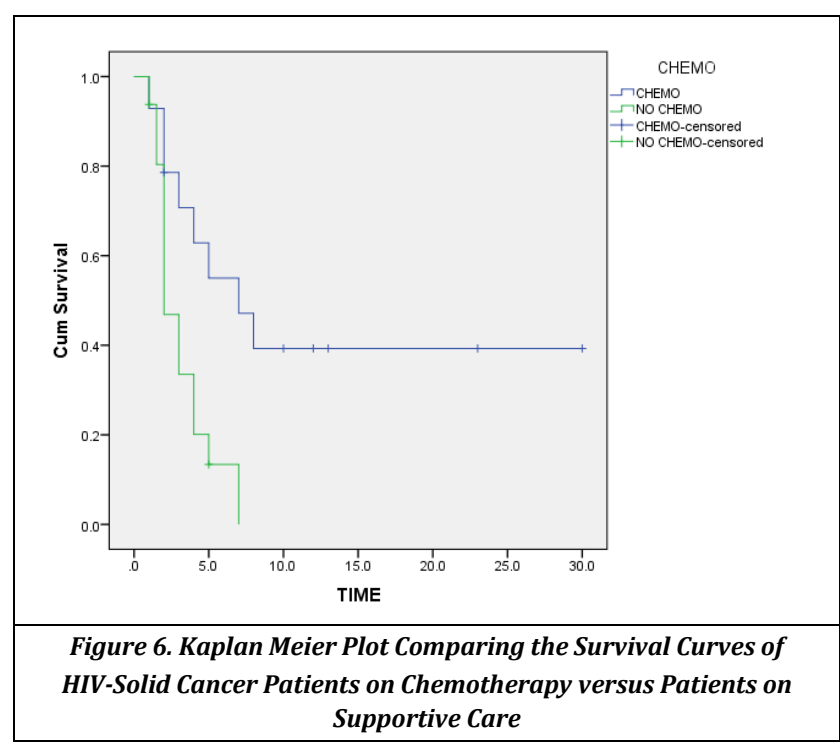

Among the Non-Hodgkin's lymphoma patients, the pathological subtype was available for twelve of them. Plasmablastic lymphoma was the most common subtype (5 cases). The other subtypes included Burkitt like lymphoma, primary CNS lymphoma, DLBCL and extranodal marginal zone lymphoma. This is depicted in Fig. 3. In the HIV patients with Non-Hodgkin`s lymphoma, extranodal presentations were more common than nodal presentations. Of the fourteen cases only three had nodal presentation whereas eleven had extranodal presentation. B symptoms (Fever, loss of weight, night sweats) were present in eight of the fourteen cases. The stage wise distribution of the lymphomas and solid tumours is shown in fig. $4 \mathrm{a}$ and $4 \mathrm{~b}$. Majority of the Non-Hodgkin`s lymphoma patients presented with stage 1 disease, while most of the solid tumours were stage 4 on presentation.

All patients were already enrolled for Anti-Retro Viral Therapy (ART), with an average CD4 count of 363. Among the 14 NHL patients, 7 received chemotherapy and had a median survival of 6 months ( $95 \%$ CI: 5.2 to 6.7 months), while the other 7 received only supportive care on account of their poor general condition and had median survival of 1.5 months (95\% CI: 0.2 to 2.7 months).The chemotherapy regimens included CHOP, CVP, R-CHOP and R-CHOP-E. 4 patients completed treatment schedule with 2 achieving partial response. The survival comparison between the patients on chemotherapy and best supportive care is shown in the following Kaplan Meier plot (Fig. 5)

Among the HIV patients with solid tumours, 47\% received chemotherapy with 5 achieving partial response (PR) and 1 achieving complete response (CR) with a median survival of 7 months as compared to 2 months in those who received only supportive care. The Kaplan Meier plot (fig.6) shows the survival curves in the chemotherapy and supportive care patients. Of the 44 cases, 11 cases were lost to follow up due to various reasons like lack of social support, difficulty in transportation and financial considerations. 


\section{DISCUSSION}

The data from UNAIDS (United Nations AIDS Organisation) indicate that there are about 36.9 million people living with HIV-AIDS as of 2017 and this number will continue to rise due to the increased lifespan conferred by HAART. In 2016, in India there were about 80,000 new cases of HIV infections and 62,000 AIDS-related deaths. There were 2.1 million people living with HIV in India, among whom $49 \%$ were receiving antiretroviral therapy.[3]

PLWHA (People Living with HIV-AIDS) are noted to be more vulnerable to malignancies than the general population. The risk is especially higher for viral mediated cancers like Kaposi's sarcoma, anogenital cancers, NHL subtypes like Burkitt's lymphoma, primary CNS lymphoma and primary effusion lymphoma. The mortality rates in PLWHA have decreased after the advent of HAART, due to lesser incidence of opportunistic infections and better immune status. Hence PLWHA live longer and experience age related cancers also at rates similar to that in non-HIV population. Even though NHLs are more common in PLWHA, Hodgkin`s lymphoma is also known to occur in cases with moderate immunosuppression rather than severe immune suppression. Hepatocellular carcinomas can occur in those that have co infection with Hepatitis B or Hepatitis C infections. Lung cancers occur with increased frequency among the smokers. Cancers in HIV infected patients tend to occur at advanced stages and are associated with worse outcomes.

Malignancies in HIV patients are a major challenge to health care providers. There are various guidelines available for the management of cancers in PLWHA, foremost among them being BHIVA (British HIV Association) guidelines. These guidelines recommend that PLWHA patients with cancer should be treated at a high-volume centre by a multidisciplinary team that includes haemato-oncologists, surgical oncologists, radiation oncologists and HIV physicians. The management includes prompt initiation of antiviral agents, anti-microbial agents against Pneumocystis carinii, mycobacterium avium intracellulare (Depending on the CD4 counts) and chemotherapy. HAART enables the use of full dose standard chemotherapy, including high dose chemotherapy and hematopoietic stem cell transplants. HIV infected patients with cancer receiving chemotherapy would also require azole antifungals, anti-herpes prophylaxis (In patients with past herpetic infections) and appropriate vaccinations.

In this study, we have documented the clinical spectrum of cancers occurring in PLWHA in South India. In the Pre HAART era, the majority of cancers in PLWHA were ADCs (Like aggressive NHL, invasive cervical cancers, Kaposi sarcoma and primary CNS lymphomas) due to their low CD4 counts and profound immunosuppression. But in the HAART era, NADCs occur more commonly than ADCs, since PLWHA live longer and hence tend to develop age related and substance abuse related cancers like the general population. ${ }^{4}$ The incidence of ADCs has declined by $70 \%$ after the introduction of HAART.[6] In this study, thirty two patients (72.7\%) had Non AIDS Defining Cancers (NADC) and twelve had AIDS defining cancers (ADC). This is higher than that reported by Dhir et al in a study involving patients with HIV and cancer in western India where NADCs accounted for $43 \% .^{[7]}$
The most common malignancy in this study was NonHodgkin`s Lymphoma (B-cell lineage), similar to other studies by Dal Maso et al[8] and Dhir et al. Extranodal presentations were more common than nodal presentations. B symptoms were present in $57 \%$ of the NHL cases. Plasmablastic lymphoma was the most common NHL subtype, comprising $36 \%$ of the NHL cases. This is in contrast to the findings in the study by Shiels et al where DLBCL (Diffuse large $\mathrm{b}$ cell lymphoma) was found to be the most common NHL in HIV patients.[9] Carcinoma penis, cervical cancers and oropharyngeal cancers which are considered to be HPV (human papilloma virus) related constituted $20.4 \%$ of the cases, suggesting a preventive role for HPV vaccination in these cases.

The mean time to presentation of cancer after the diagnosis of HIV was 5.4 years and hence PLWHA have to be under prolonged surveillance for cancer. The mean BMI was 19.45 and about $52.2 \%$ had a BMI $<20$ with recent weight loss $>5 \%$ of their body weight, thus placing them in the cachectic category. ${ }^{[10]}$ This highlights the need to address the nutritional deficiencies in these patients who often have significant malnutrition due to the HIV, cancer, infections and poor social support. Nearly $29.5 \%$ had a poor performance status (ECOG scale 3/4), thus precluding upfront chemotherapy. All patients were already enrolled on antiretroviral therapy. Early initiation of ART results in better quality of life and better performance status, thus enabling use of more intensive chemotherapy regimens.[11] None of the patients in this study had significant drug interactions involving ART and chemotherapy.

Among NHL cases, early stage presentations were common (42\% with stage 1) while solid cancer cases presented at advanced stages ( $50 \%$ with stage 4 disease). The overall mean CD4 count was 363. The mean CD4 counts among the NHL and solid cancers were 274 and 439 respectively. Among the fourteen NHL patients, seven (50\%) received chemotherapy (like CHOP, CVP, R-CHOP and RCHOP-E) and had a median survival of 6 months (95\% CI: 5.2 to 6.7 months), while the other seven received only supportive care on account of their poor general condition and had a median survival of 1.5 months (95\% CI: 0.2 to 2.7 months). Fourteen of the thirty cases of HIV with solid tumours received chemotherapy with five achieving partial response (PR) and one achieving complete response (CR) with a median survival of seven months as compared to two months in those who received only supportive care. There was a response rate of $46 \%$ with standard chemotherapy in HIV patients with solid cancers. This suggests that full dose chemotherapy is feasible in $50 \%$ of patients with HIV-NHL and $46 \%$ of HIV patients with solid cancers, partly due to the improvement in performance status provided by HAART.[11] This study was a single centre study with a small sample size. Larger multicentric studies may throw more light on the cancer patterns and response to therapy in these PLWHA patients.

\section{CONCLUSIONS}

In the HAART era, NADCs are more common than ADCs. The most common malignancy in HIV patients is NHL, with 
plasmablastic subtype being the commonest in this study. Extranodal presentations are more common than nodal presentation. Standard chemotherapy achieves a response rate of $50 \%$ in HIV-NHL cases and $46 \%$ in HIV patients with solid cancers. The study illustrates the need for prolonged surveillance for the development of cancer in HIV patients. Early initiation of HAART, optimal chemotherapeutic regimens, appropriate palliative care and nutritional support should form an integral part of the care of HIV patients with cancer.

\section{REFERENCES}

[1] Paranjape RS, Challacombe SJ. HIV/AIDS in India: an overview of the Indian epidemic. Oral Dis 2016;(22 Suppl 1):10-4.

[2] Silverberg MJ, Chao C, Leyden WA, et al. HIV infection and the risk of cancers with and without a known infectious cause. AIDS 2009;23(17):2337-45.

[3] https://www.who.int/news-room/fact-heets/detail/hivaids.

[4] Yarchoan R, Tosato G, Little RF. Therapy insight: AIDSrelated malignancies-the influence of antiviral therapy on pathogenesis and management. Nat Clin Pract Oncol 2005;2(8):406-15.

[5] Powles T, Robinson D, Stebbing J, et al. Highly active antiretroviral therapy and the incidence of non-AIDSdefining cancers in people with HIV infection. Journal of Clinical Oncology 2009;27(6):884-90.

[6] Shiels MS, Pfeiffer RM, Gail MH, et al. Cancer burden in the HIV-infected population in the United States. Journal of the National Cancer Institute 2011;103(9):753-62.

[7] Dhir AA, Sawant S, Dikshit RP, et al. Spectrum of HIV/AIDS related cancers in India. Cancer Causes Control 2008;19(2):147-53.

[8] Dal Maso L, Serraino D, Franceschi S. Epidemiology of AIDS-related tumours in developed and developing countries. Eur J Cancer 2001;37(10):1188-201.

[9] Shiels MS, Pfeiffer RM, Hall HI, et al. Proportions of Kaposi sarcoma, selected non-Hodgkin lymphomas, and cervical cancer in the United States occurring in persons with AIDS, 1980-2007. J Am Med Assoc 2011;305(14):1450-9.

[10] Fearon K, Strasser F, Anker SD, et al. Definition and classification of cancer cachexia: an international consensus. Lancet Oncology 2011;12(5):489-95.

[11] Noy A. Optimizing treatment of HIV-associated lymphoma. Blood 2019;134(17):1385-94. 